\title{
Article \\ Effect of total starch and resistant starch in commercial extruded dog foods on gastric emptying in non-racing sled dogs
}

\author{
Taylor L. Richards ${ }^{1}$, Alexandra Rankovic ${ }^{2}$, John P. Cant ${ }^{1}$, Anna K. Shoveller ${ }^{1}$, Jennifer L. Adolphe ${ }^{3}$, Dan Ramdath ${ }^{4}$, \\ Adronie Verbrugghe ${ }^{2}$
}

${ }^{1}$ Centre for Nutrition Modeling, Department of Animal Biosciences, University of Guelph, 50 Stone Road East, Guelph, Ontario, N1G 2W1, Canada; tricha16@uoguelph.ca; jcant@uoguelph.ca; ashovell@uoguelph.ca

${ }^{2}$ Department of Clinical Studies, Ontario Veterinary College, University of Guelph, 50 Stone Road East, Guelph, Ontario, N1G 2W1, Canada; arankovi@uoguelph.ca; averbrug@uoguelph.ca

${ }^{3}$ Veterinary Biomedical Sciences, University of Saskatchewan, Saskatoon, SK S7N 5B4, Canada; Jennifer.Adolphe@adm.com

${ }^{4}$ Guelph Research and Development Centre, Agriculture and Agri-Food Canada, 93 Stone Road West, Guelph, Ontario, N1G 5C9, Canada; dramdath@uoguelph.ca

*Correspondence: averbrug@uoguelph.ca

Simple Summary: Gastric emptying is the release of nutrients from the stomach into the small intestine. The rate at which gastric emptying occurs may be associated with diabetes and obesity risk in humans and could help prevent weight gain in dogs. The largest portion of carbohydrates in pet diets is provided by various starches that are digested and absorbed at different rates. This study investigated the effects of common starch ingredients found in commercial dog foods on the gastric emptying rate in dogs. Dogs received each test diet once (4 total) and a glucose control twice in a randomized order, along with acetaminophen. Blood samples were taken once prior to meal consumption and at multiple time points after to determine acetaminophen concentrations. A mathematical model was used to estimate the rate of gastric emptying using post-prandial acetaminophen concentrations. Overall, more gastric emptying occurred at a faster rate in dogs when fed the diet containing the highest fraction of starch ingredients resistant to canine digestion. These findings suggest that the inclusion of different starch sources may be associated with altered digestion and absorption of nutrients, which consequently effects gastric emptying rate. This research may provide a mechanism to prevent weight gain in dogs.

Abstract: Gastric emptying rate (GER) may impact diabetes and obesity in humans and could provide a method to reduce canine weight gain. Starch, the most common source of carbohydrates (CHOs) in pet food, is classified as rapidly or slowly digestible, or resistant to digestion. This study investigated starch source effects in commercial extruded dog foods on the GER of 11 healthy adult Siberian Huskies (5.63 \pm 0.72 years; mean \pm SEM). Test diets were classified as traditional, grain-free, whole-grain, and vegan. Dogs received each diet once, a glucose control twice, and acetaminophen (Ac) as a marker for GER in a randomized, partially-replicated, 6x6 Latin square design. Pre- and post-prandial blood samples were collected at 16 timepoints from -15 to 480 minutes. Serum Ac concentrations were assessed via standard spectrophotometric assays and fitted with a mathematical model to estimate parameters of GER. Data was analyzed using a repeated measures ANOVA, followed by a Tukey-Kramer post-hoc test when significant $(p<0.05)$. More total emptying $(p=0.0430)$ occurred at faster rate $(\mathrm{p}=0.0668)$ in dogs fed the grain-free diet, which contained the lowest total starch $(34.03 \pm 0.23 \%)$ and highest resistant starch $(0.52 \pm 0.007 \%)$. This research may benefit future diet formulations to reduce the prevalence of canine weight gain.

Keywords: canine, carbohydrates, grain-free, pet food, pharmacokinetic mathematical modelling, acetaminophen tracking.

\section{Introduction}

Obesity is the most common nutrition-related disorder facing the domestic dog population today and can affect quality and longevity of life $[1,2]$. Obesity can be described as the excess accumulation of adipose tissue within the body and is usually the result of either excessive dietary intake or inadequate energy utilization, which leads to a state of positive energy balance [4]. Increased risk and aggravation of numerous co- 
morbidities, including cardiac, respiratory and orthopedic diseases, cancer and diabetes mellitus, have been associated with this disorder [3].

The rate of gastric emptying (GE) and the concentrations of post-prandial glucose (PPG), which are directly associated in humans, may contribute to the onset of obesity and its secondary complications in canines [5,6]. Gastric emptying rate (GER) is defined as the speed with which nutrients are released from the stomach into the duodenum of the small intestine following ingestion [7]. A faster GER results in higher PPG concentrations [5]. If PPG concentrations exceed the capacity of insulin to clear glucose into the tissues through insulin dependent glucose transporters, this may lead to lipogenesis, which will ultimately contribute to weight gain and increased risk for obesity [5]. Although this relationship has predominantly been observed in human and rat models, a similar relationship is thought to exist in dogs $[5,6]$ and was the impetus for the present study.

Carbohydrates (CHOs) are the most prevalent macronutrient found in dry extruded pet foods ( $40-60 \%$ dry matter) $[10,11]$. The largest proportion of CHOs in pet food is provided by starch [12], which is made up of two glucose polymers: amylose and amylopectin [13]. Amylose is less susceptible to digestion due to its linear structure while amylopectin has a branched chain structure, making it more susceptible to digestion [13]. The ratio of amylose to amylopectin varies across starch sources; most starch sources contain 20-35\% amylose [14-16], while the amylose content of legume starches ranges from 29-65\% [15-17]. Due to the differences in proportion of amylose, starch sources can be classified based on their rate of digestion: rapidly digestible; slowly digestible; or resistant to digestion [10]. Resistant starch (RS) refers to the portion of starch that cannot be enzymatically digested and enters the large intestine intact where it is fermented by microorganisms [18]. The glycemic index (GI) was developed as a way to rank $\mathrm{CHOs}$ based on their acute post-prandial glycemic response in comparison to a control food (white bread or a standard glucose solution) [19]. More RS is hypothesized to result in lower PPG and therefore lower GI values due to the less digestible nature of this starch. In comparison, less RS means more digestible starch, which results in an increase in PPG and therefore GI. Due to the differences in proportion of these three starch fractions within foods and their subsequent variation in rate and degree of digestibility, the effects of CHOs in pet food on GER, PPG and GI are expected to vary.

In order to assess the rate of GE, several approaches have been investigated. Gamma Scintigraphy (GS) is considered by many to be the "gold standard" method to measure GE; however, this approach is fairly expensive and involves radiation exposure [7]. Acetaminophen (Ac) tracking is a method based on the understanding that Ac is slowly absorbed from the stomach but rapidly absorbed in the small intestine [20]. This approach is significantly less expensive than GS and involves no radiation exposure [7]. Additionally, a study completed by Glerup et al. in 2007 compared three different methods used to assess GE in research animals, including GS, Ac tracking and the ${ }^{13} \mathrm{C}$-acetate breath test, and found that the results obtained from Ac tracking were similar to the results obtained via GS [21]. Consequently, Ac tracking is considered a reliable method to assess GER in mammals and was used in the present study [22].

Overall, the current literature discussed above suggests a link between the type of CHO consumed, its resulting GER and subsequent GI and PPG. To the authors knowledge, there is a dearth of research on this topic using a dog model. Determining the effects of CHOs in pet foods on GER and, in turn, PPG and GI values may be paramount in preventing weight gain and ultimately reducing the prevalence of conditions like obesity in dogs. This study aimed to elucidate the relationship between starch sources found in various commercial extruded dog diets and the GER in healthy adult Siberian Huskies. Based on the aforementioned studies, authors hypothesized that the presence of digestible starch in the diet will increase PPG, GI and GER whereas RS will decrease PPG, GI and GER. Rankovic et al. (2020) measured the PPG and GI values of the diets used in the present study and found that the order of GI from highest to lowest was: traditional grain 
diet $>$ vegan diet $>$ whole grain diet $>$ grain-free diet [23]. Although numerical differences were observed, there were no significant differences in GI between dietary treatments [23]. The same study found that the grain-free diet induced the lowest area under the PPG curve during 30-480 minutes post-prandial compared to the other dietary treatments, although these results were not significant [23]. Therefore, it was predicted that the grain-free diet, which results in the lowest PPG and GI values, should induce the slowest GER.

\section{Materials and Methods}

Animals: The experimental protocol for this research was approved by the University of Guelph Animal Care Committee (AUP\#3650) and in accordance with national and institutional guidelines for the care and use of animals. Eleven adult, clientowned Siberian Husky dogs $(n=4$, neutered males; $n=5$, spayed females; $n=2$, intact females) were used in this study. Dogs were housed at an off-site facility (Rajenn Siberian Huskies, Ayr, ON) that had been visited and approved by the University of Guelph's Animal Care Services. The dogs had a mean age of $5.63 \pm 0.72$ years (range 1.00-10.67 years) and a mean body weight (BW) of $23.32 \pm 1.15 \mathrm{~kg}$ (range 19.00-30.68 kg). Body condition scores (BCS) for the dogs ranged between 3 and 6 (mean \pm SD: $4.91 \pm 0.63$ ) on a 9-pointscale [24]. All dogs were deemed healthy based on medical history, physical examination, complete blood count (CBC) and serum biochemistry profile. Dogs were excluded if they had received medications 6 months prior to enrolment, had abnormalities on their physical examination, CBC or serum biochemistry, or were younger than one year of age. The dogs were housed together in a group housing system and were separated and handled individually on study days. All dogs were transitioned onto the same diet (GO! FIT + FREE Adult Dog Food, Petcurean Pet Nutrition, Chilliwack, BC, CA) 2 months prior to the start of the investigation. Dogs continued to eat this background diet throughout the entire study period. Dogs were fed the background diet in amounts suitable to maintain BW based on their dietary history. Body weight and BCS were recorded at each study visit and the amount fed was adjusted to maintain BW as required.

Dietary Treatments: : Four commercially available dog diets were used as the test food in this study: Purina ${ }^{\circledR}$ Dog Chow $^{\circledR}$ (Nestle Purina Petcare, St. Louis, MO, USA) as the traditional diet, GO! SENSITIVITY + SHINETM Limited Ingredient Duck Recipe (Petcurean Pet Nutrition, Chilliwack, BC, CA) as the grain-free diet, SUMMIT TM Three Meat Adult Recipe (Petcurean Pet Nutrition, Chilliwack, BC, CA) as the whole-grain diet and Natural Balance ${ }^{\circledR}$ Vegetarian Formula (Dick Van Patten's Natural Balance Pet Foods, Burbank, CA, USA) as the vegan diet. Three diets were classified based on the main starch sources that were listed on their ingredient panels: traditional grain (corn, wheat), whole grains (oats, rye), grain-free (peas, lentils); the fourth diet vegan had no animal ingredients (Table 1). A 50\% (wt/vol) glucose solution was used as the control.

The proximate analyses of the four commercial diets were performed by Central Testing Laboratories Ltd. (Winnipeg, MB, CA) (Table 1). Proximate analyses of the diets were performed as follows, according to the Association of Official Analytical Chemists (AOAC) and the American Oil Chemist Society (AOCS) methods: ash by gravimetry (AOAC 923.03), crude protein by combustion (AOAC 990.03), crude fat by extraction (AOCS Am 5-04), crude fiber by gravimetry (AOCS Ba6a-05), total dietary fiber (TDF) by gravimetry (AOAC 991.43, 985.29) and moisture by gravimetry (AOAC 930.15).

Each dog received an amount of each dietary treatment that provided 25 grams of available $\mathrm{CHO}(\mathrm{AvCHO})$ (62 $\mathrm{g}$ of the traditional diet, $77 \mathrm{~g}$ of the whole-grain diet, $65 \mathrm{~g}$ of the grain-free diet, and $55 \mathrm{~g}$ of the vegan diet), as determined by free sugar and starch analysis (Table 2). The quantity of $\mathrm{AvCHO}$ within the food was determined directly using the following equation (Food and Agriculture Organization of the United Nations 2003): 
Av $\mathrm{CHO}=$ weight in grams [monosaccharides+disaccharides+oligosaccharides+[polysaccharides-fiber]].

The total starch (TS) and RS content of each diet were determined enzymatically using commercially available assay kits (Megazyme International, Wicklow, Ireland; AOAC Method 996.11 and 2002.02, respectively) (Table 1). Free sugar content was determined via High Performance Liquid Chromatography (HPLC) as previously described by Brummer et al. (2015) (Table 1) [27]. The GI values of the traditional, whole grain, grain-free and vegan diets, as reported previously by Rankovic et al. (2020), were $77 \pm 18,61 \pm 7,50 \pm 13$ and $71 \pm 17$, respectively.

Table 1. Proximate analysis, total dietary fibre and energy contents as well as in vitro starch and free sugar contents of four commercial extruded dog foods containing different starch sources fed in a gastric emptying trial to 11 clientowned Siberian Huskies.

Treatment

\begin{tabular}{lcccc} 
& Traditional & Whole grain & Grain-free & Vegan \\
\hline Moisture, \% & 5.85 & 6.32 & 6.56 & 7.15 \\
Crude Protein, \%DM & 25.55 & 26.10 & 28.59 & 23.46 \\
Crude Fat, \%DM & 10.25 & 10.21 & 11.58 & 8.20 \\
Ash, \%DM & 7.24 & 10.10 & 7.66 & 5.00 \\
Crude Fiber, \%DM & 1.05 & 1.24 & 3.14 & 2.10 \\
NFE1, \%DM & 55.91 & 52.35 & 49.03 & 61.24 \\
TDF, \%DM & 8.10 & 8.80 & 10.3 & 10.0 \\
GE, kcal/kgDM ${ }^{2}$ & 4712 & 4593 & 4728 & 4619 \\
ME, kcal/kgDM & 3959 & 3841 & 3816 & 3846 \\
AvCHO, \%DM & 42.51 & 41.13 & 34.63 & 48.52 \\
Total Starch, \%DM & $41.69 \pm 1.48$ & $40.83 \pm 1.90$ & $33.91 \pm 0.44$ & $48.06 \pm 4.26$ \\
Resistant Starch, \%DM & $0.41 \pm 0.02$ & $0.16 \pm 0.01$ & $0.56 \pm 0.02$ & $0.26 \pm 0.03$ \\
Glucose, \%DM & $0.099 \pm 0.0032$ & $0.023 \pm 0.0008$ & $0.032 \pm 0.0013$ & $0.029 \pm 0.0018$ \\
Sucrose, \%DM & $1.40 \pm 0.027$ & $0.56 \pm 0.028$ & $1.38 \pm 0.022$ & $0.87 \pm 0.036$
\end{tabular}

DM, dry matter; NFE, nitrogen free extract; TDF, total dietary fiber; GE, gross energy; ME, metabolizable energy;

AvCHO, available carbohydrates

Values reported on dry matter basis, except for moisture.

${ }^{1} \mathrm{NFE}=100-$ [weight in grams [protein + fat + ash + crude fiber] in $100 \mathrm{~g}$ of food]

${ }^{2} \mathrm{GE}(\mathrm{kcal} / \mathrm{kgDM})=(5.7 \times \mathrm{g}$ protein $)+(9.4 \times \mathrm{g}$ fat $)+[4.1 \times(\mathrm{g}$ NFE $+\mathrm{g}$ crude fiber $)][25]$

${ }^{3} \mathrm{ME}(\mathrm{kcal} / \mathrm{kgDM})=575+[0.816 \times \mathrm{GE}(\mathrm{kcal} / \mathrm{kg})]+(12.08 \times \%$ fat $)-(52.76 \times \%$ crude fiber $)-(20.61 \times \%$ protein $)-(6.07 \times$

$\%$ moisture) [26]

Ingredient panels, starch sources in italic:

Traditional: Whole grain corn, meat and bone meal, corn gluten meal, beef fat preserved with mixed-tocopherols, soybean meal, poultry by-product meal, chicken, egg and chicken flavour, whole grain wheat, pork \& poultry digest, salt, calcium carbonate, potassium chloride, mono and dicalcium phosphate, choline chloride. Vitamins [vitamin e supplement, niacin (vitamin b-3), vitamin a supplement, calcium pantothenate (vitamin b-5), pyridoxine hydrochloride (vitamin b-6), vitamin b-12 supplement, thiamine mononitrate (vitamin b-1), vitamin d-3 supplement, riboflavin supplement (vitamin b-2), menadione sodium bisulfite complex (vitamin k), folic acid (vitamin b-9), biotin (vitamin b-7)] Minerals [zinc sulphate, ferrous sulphate, manganese sulphate, copper sulphate, calcium iodate, sodium selenite] 1lysine monohydrochloride yellow 6 , yellow 5, red 40, soybean oil, blue 2 , garlic oil. 
Whole grain: Chicken meal, oatmeal, whole brown rice, rye, barley, chicken fat (preserved with mixed tocopherols), salmon meal, lamb meal, natural chicken flavour, whole dried egg, rice bran, dried kelp, flaxseed, dicalcium phosphate, calcium carbonate, potassium chloride, choline chloride, l-lysine, sodium chloride. Vitamins [vitamin a supplement, vitamin d-3 supplement, vitamin e supplement, niacin, 1-ascorbyl-2-polyphosphate (a source of vitamin c), dcalcium pantothenate, thiamine mononitrate, beta-carotene, riboflavin, pyridoxine hydrochloride, folic acid, biotin, vitamin b-12 supplement] Minerals [zinc proteinate, iron proteinate, copper proteinate, zinc oxide, manganese proteinate, copper sulphate, ferrous sulphate, calcium iodate, manganous oxide, selenium yeast] taurine, dl-methionine, dried rosemary.

Grain-free: De-boned duck, duck meal, peas, lentils, tapioca, pea flour, canola oil (preserved with mixed tocopherols), chickpeas, natural flavour, coconut oil (preserved with mixed tocopherols), monocalcium phosphate, salmon oil, sodium chloride, calcium carbonate, potassium chloride, dried chicory root, choline chloride. Vitamins [vitamin a supplement, vitamin d-3 supplement, vitamin e supplement, niacin, 1-ascorbyl-2-polyphosphate (a source of vitamin c), dcalcium pantothenate, thiamine mononitrate, beta-carotene, riboflavin, pyridoxine hydrochloride, folic acid, biotin, vitamin b-12 supplement] Minerals [zinc proteinate, iron proteinate, copper proteinate, zinc oxide, manganese proteinate, copper sulphate, ferrous sulphate, calcium iodate, manganous oxide, selenium yeast] taurine, dried rosemary. Vegan: Brown rice, oat groats, barley, peas, potato protein, canola oil (preserved with mixed tocopherols), potatoes, dicalcium phosphate, dried tomato pomace, natural flavor, calcium carbonate, potassium chloride, choline chloride, taurine. Vitamins [vitamin e supplement, vitamin a supplement, d-calcium pantothenate, niacin, riboflavin supplement, vitamin d-2 supplement, vitamin b-12 supplement, thiamine mononitrate, pyridoxine hydrochloride, folic acid, biotin] Minerals [zinc proteinate, zinc sulfate, ferrous sulfate, iron proteinate, copper sulfate, copper proteinate, manganese sulfate, manganese proteinate, calcium iodate, sodium selenite] salt, flaxseed, dried spinach, parsley, cranberries, 1lysine monohydrochloride, 1-carnitine, citric acid (used as a preservative), mixed tocopherols (used as a preservative), yucca schidigera extract, dried kelp, 1-ascorbyl-2-polyphosphate (source of vitamin c), rosemary extract.

Table 2. Macronutrient content of four commercial extruded dog foods containing different starch sources fed in a meal response test to 11 client-owned Siberian huskies, expressed as the dry-matter quantity in grams that each dog received to provide $25 \mathrm{~g}$ available carbohydrate of each diet.

Treatment

\begin{tabular}{lcccc} 
& Traditional & Whole grain & Grain-free & Vegan \\
\hline Portion size for 25g AvCHO (g) & 62 & 77 & 65 & 55 \\
Crude Protein, (g) & 15.84 & 20.09 & 18.58 & 15.46 \\
Crude Fat, (g) & 6.35 & 7.86 & 7.52 & 5.41 \\
Crude Fiber, (g) & 0.651 & 0.954 & 2.04 & 1.15 \\
TDF, (g) & 5.02 & 6.77 & 6.69 & 5.50
\end{tabular}

AvCHO, available carbohydrates; TDF, total dietary fiber;

Values reported on dry matter basis.

Study Design: This study was conducted according to a partially replicated 6x6 Latin square design where each dog received each commercial diet once and a $50 \%$ (wt/vol) glucose solution as a control that each dog received twice. Between trials there was a 7-day washout period during which only the background diet was fed.

Dogs were fasted overnight $(14 \mathrm{~h})$ prior to each test. On test days, dogs were weighed and BCS was recorded. Emla cream (2.5\% Lidocaine) was applied to the dogs' legs at the site were a 20 Ga IV catheter (Insyte-W 20GA × 1.1, Becton Dickinson, Franklin Lakes, NJ, USA) was placed into a cephalic or saphenous vein. Once placed, catheters were flushed immediately with $2 \mathrm{~mL}$ of $0.9 \%$ sodium chloride solution (Baxter International, Deerfield, IL, USA), followed by $0.1 \mathrm{~mL}$ of $4.0 \%$ sodium citrate (Baxter International, Deerfield, IL, USA) to prevent clotting. Prior to blood collection, catheters were flushed with $0.5 \mathrm{~mL}$ sterile isotonic sodium chloride solution, and 0.5 
$\mathrm{mL}$ of blood was withdrawn and discarded to avoid dilution. Dogs were allowed a minimum of $15 \mathrm{~min}$ to recover from catherization. The first blood sample occurred at 15 minutes (baseline) prior to feeding the test diet or glucose control with the oral dose of Ac ( $16.30 \mathrm{mg} / \mathrm{kg} \mathrm{BW})$ administered immediately following feeding. Timing began as soon as the dog began ingesting the test diet or glucose control and blood samples were taken at 15, 30, 45, 60, 90, 120, 150, 180, 210, 240, 270, 300, 360, 420 and 480 minutes post-prandial. At each timepoint $2.5 \mathrm{ml}$ of blood was collected into serum separation tubes (Vacutainer ${ }^{\mathrm{TM}}$, Becton Dickinson, Franklin Lakes, NJ, USA) and immediately refrigerated $\left(4^{\circ} \mathrm{C}\right)$. After collection, catheters were flushed with $2 \mathrm{~mL}$ of sodium chloride and $0.1 \mathrm{~mL}$ of sodium citrate to maintain patency. The samples were centrifuged at $4^{\circ} \mathrm{C}$ at $1000 \mathrm{~g}$ for 10 minutes (Legend RT, Kendro Laboratory Products, Asheville, NC, USA), aliquoted and serum was stored in a freezer at $-20^{\circ} \mathrm{C}$ until analysis.

Acetaminophen Assay and Gastric Emptying Model: Serum Ac content was determined using a commercially available spectrophotometric assay kit (Paracetamol Assay Kit K8002, Cambridge Life Sciences, Cambridgeshire, UK). The assay was performed in triplicate according to the manufacturer's instructions and run on a Powerwave Xs Microplate Spectrophotometer (Biotek, Winooski Vermont, USA) with KC4 Data Analysis Software (Biotek, Winooski Vermont, USA) (Wavelength $=650 \mathrm{~nm}$ ).

The mathematical model used to estimate GE parameters from the serum Ac time course has been described previously by Stahel et al. (2016) [20]. Briefly, Ac is assumed to enter serum by first-order GE according to rate constant $\mathrm{ksB}$, and exit serum according to rate constant $\mathrm{kel}_{\mathrm{el}}$ to yield the following differential equation:

$$
\mathrm{dAcse} / \mathrm{dt}=\mathrm{ksB} \times \mathrm{Acst}-\mathrm{kel}_{\mathrm{el}} \times \mathrm{Acse}
$$

where Acst and Acse are the masses of Ac (mg) in stomach and serum, respectively. For each sampling interval, $\mathrm{ksB}_{\mathrm{sB}}$ is assigned a value of 0 , $\mathrm{ksB2}_{\mathrm{s} 2}$ or ksB3, representing no, slow or fast emptying, respectively, based on the observed rate of change in serum Ac concentration $(\Delta \mathrm{cAcse})$. Accordingly, $\mathrm{k}_{\mathrm{sB}}=0$ when $\Delta \mathrm{cAcse},<-0.05 \mathrm{mg} / \mathrm{L} / \mathrm{min}, \mathrm{k}_{\mathrm{SB}}=\mathrm{ksB}_{\mathrm{SB}}$ when $-0.05 \mathrm{mg} / \mathrm{L} / \mathrm{min} \leq \Delta \mathrm{cAcse} \leq 0.05 \mathrm{mg} / \mathrm{L} / \mathrm{min}$, and $\mathrm{ksB}=\mathrm{ksB}_{3}$ when $\Delta \mathrm{cAcse}>0.05$. The predicted concentration of Ac in serum (cAcse) was calculated from Acse assuming a volume of distribution of $50 \%$ of BW. Best-fit values of $\mathrm{ksB}, 2,_{\mathrm{ksB}, 3}$ and kel were estimated with the Solver function of Microsoft Office Excel 2020 Version 16.37 to minimize residual sums of squares between predicted and observed cAcse above baseline. Goodness of fit for each curve is presented as the root mean square prediction error (rMSPE) as a percentage of the mean observed:

$$
r M S P E \%=\frac{\sqrt{\sum_{i=1}^{n}\left(\text { predicted }_{i}-\text { observed }_{i}\right)^{2} / n}}{\sum_{i=1}^{n} \text { observed }_{i} / n}
$$

where $\mathrm{n}$ is the number of observations in the time course. Sums were calculated of the amount of time spent emptying rapidly (time-fast) and slowly (time-slow) as well as the amount of time with no emptying (time-off). A total emptying index to amalgamate time and rate of GE was calculated as ksB2 $\times$ time-slow $+\mathrm{ksB3}_{\text {SB }} \times$ time-fast.

Statistical Analysis: Data were analyzed using SAS (Statistical Analysis System, Version 9.4, Cary, NC, USA) software. Curves with residual sum of squares values greater than $200 \mathrm{mg}^{2} / \mathrm{L}^{2}$ were deemed unfit and removed from analysis (17 total: glucose control, 2; traditional, 3; grain-free, 4; whole-grain, 3; vegan, 5). Figure 1 
below provides an example of predicted and observed $\Delta$ cAcse curves used in this study for one dog on one treatment. Repeated measures analysis of variance (ANOVA) followed by a Tukey-Kramer post-hoc test was used to compare treatment means. Treatment was used as the fixed effect, and time was included as the repeated term with dog as the experimental unit. A $\mathrm{p}<0.05$ was considered significant and $\mathrm{p}<$ 0.10 was considered a trend.

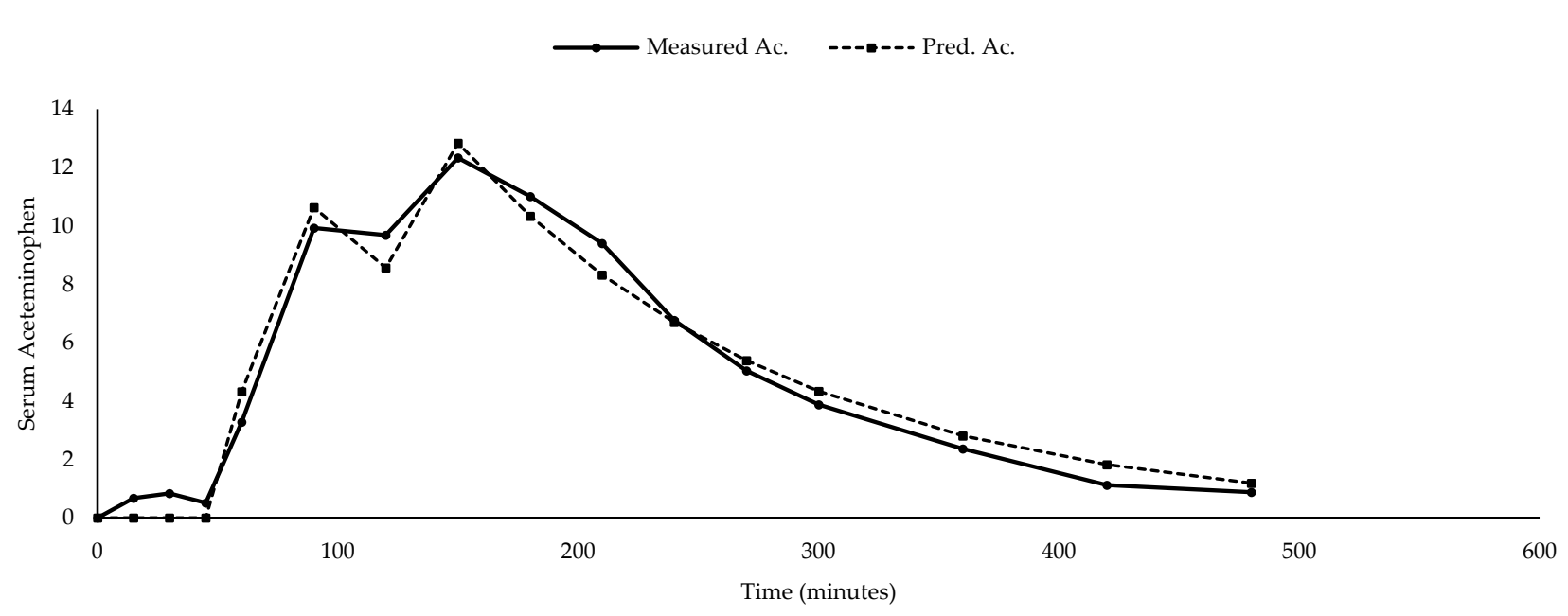

Figure 1. Predicted and measured serum Acetaminophen concentration curves in a gastric emptying study using Acetaminophen as a tracer in dogs over a 480-minute period. Predicted serum Acetaminophen concentrations were determined using a mathematical model, described previously by Stahel et al. [20].

\section{Results}

All statistical analyses values discussed below are displayed in Table 3.

First-order slow gastric emptying rate constant $\left(\boldsymbol{k}_{s B 2}\right)$ : There was an effect of treatment $(p=0.046)$ in that the grain-free diet induced higher ksB2 values compared to both the traditional diet $(p=0.023)$ and glucose control $(p=0.012)$. Additionally, a trend was observed in that the grain-free diet induced greater $\mathrm{ksB}_{\mathrm{s} 2}$ values as compared to the vegan diet $(p=0.0903)$ and lower when fed the traditional diet compared to the wholegrain diet $(\mathrm{p}=0.0724)$.

First-order fast gastric emptying rate constant $\left(k_{S B 3}\right)$ : A trend was observed in the effect of treatment on $\mathrm{kSB}_{\mathrm{SB}}(\mathrm{p}=0.066)$.

First-order elimination constant $\left(K_{e l}\right)$ : A trend was observed in the effect of treatment on $k_{e l}(p=0.056)$.

Area under the Acetaminophen Curve: There was an effect of treatment $(p=0.040)$ on the area under the Ac curve (AUC). The AUC was higher following consumption of the grain-free diet compared to the glucose control $(p=0.0042)$. Additionally, a trend was observed in that the whole-grain diet induced a greater AUC compared to the glucose control $(p=0.0736)$.

Total Emptying Index: There was an effect of treatment on total emptying $(\mathrm{p}=0.043)$. Total emptying was greater in dogs when fed the grain-free diet in contrast to the traditional diet $(p=0.0309)$ and glucose control $(p=0.0106)$. Two trends were 
observed: total emptying was greater following ingestion of the grain-free vs. vegan $\operatorname{diet}(\mathrm{p}=0.0814)$ and whole-grain vs. traditional diet $(\mathrm{p}=0.0828)$.

Time-off, Time-slow, Time-fast: There was no effect of treatment on time-off, time-slow or time-fast values $(\mathrm{p}=0.493, \mathrm{p}=0.740, \mathrm{p}=0.704$, respectively).

Table 3. Markers of gastric emptying, via acetaminophen tracking, in a meal response test in 11 client-owned Siberian Huskies fed four commercial extruded dog foods containing different starch sources in amounts to provide $25 \mathrm{~g}$ available carbohydrate of each diet.

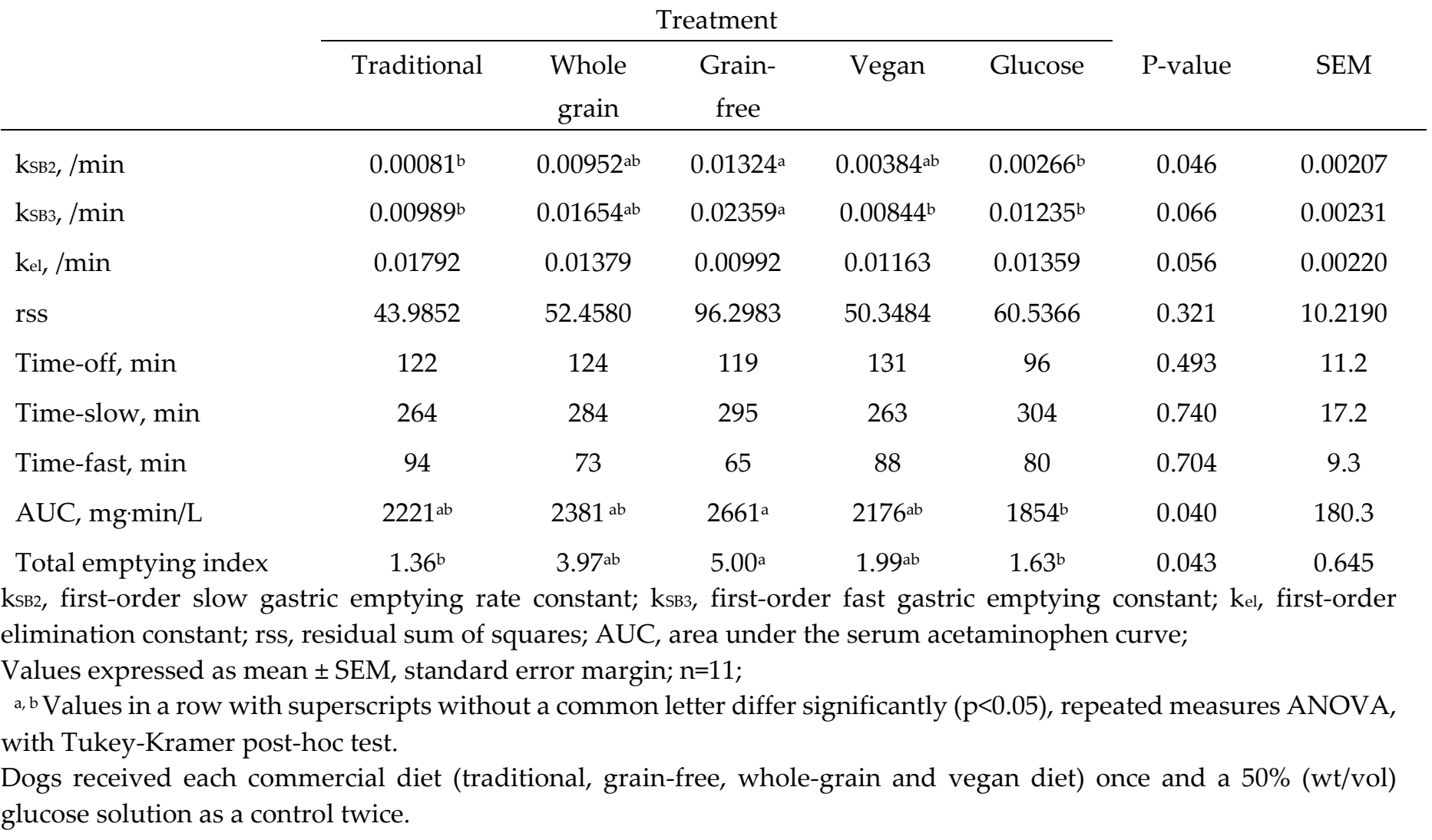

\section{Discussion}

The results presented herein suggest that the grain-free diet, which included peas and lentils as the primary starch sources, induced both the greatest amount of GE and the most rapid GER. These findings may be attributed to the proportional differences between slowly digestible, rapidly digestible and RS in the test diets.

In human trials, pulse ingredients, like lentils, peas and chickpeas, significantly attenuate PPG and are consequently categorized as low GI foods [28]. Pulse ingredients contain higher amounts of slowly digestible starch (SDS) and RS, and lower amounts of rapidly digestible starch (RDS) compared to cereal grains, which contain lower concentrations of SDS and RS and higher concentrations of RDS [28]. The relative proportions of SDS, RDS and RS in pulse ingredients have been attributed to their high amylose content compared to cereal starches, strong interactions between amylose chains, the presence of intact cell structures after cooking, high dietary fiber components and anti-nutrients [28]. The differences in starch profiles are thought to subsequently result in lower rates of hydrolysis, PPG and GI following ingestion of pulses compared to cereal grains [28]. The proportions of SDS, RDS and RS may influence the GER of a diet as well, as SDS slows GE [29]. Taken together, diets with the more pulse ingredients, thereby containing the greatest proportion of SDS, RS and lowest proportion of RDS, should result in the lowest PPG, GI and GER. 
In the present study, the grain-free diet contained the highest amount of RS and lowest amount of TS relative to the other diets. Furthermore, the grain-free diet contained the most pulse ingredients, indicating that it also contained the highest amount of SDS and least amount of RDS. In comparison, the vegan, traditional and whole-grain diets had less RS, more TS, and less pulses ingredients, indicating less SDS and more RDS. Based on these findings, it was hypothesized that the grain-free diet should result in the lowest PPG, GI and GER due to the higher amount of pulse ingredients.

Rankovic et al. (2020) indeed found that the grain-free diet used in the present study had the lowest GI value compared to the traditional, whole-grain and vegan diets, although these results were not significantly different [23]. The same study found that the grainfree diet induced the lowest area under the PPG curve between 30-480 minutes compared to the other dietary treatments [23]. The lower PPG concentration induced by the grain-free diet was only significant when compared to the whole grain diet and the glucose control between 0-150 minutes [23]. However, contrary to the authors hypothesis for the present study, the grain-free diet, which contained the highest concentration of RS and induced the lowest GI and PPG values, resulted in the fastest GER.

One possibility for these contradictory results in dogs may be due to the effect that RS has on the osmoreceptors found in the duodenum [8,9]. Gastric emptying is thought to be slowed when these receptors are stimulated by material arriving from the stomach, specifically by the products of starch (sugars) and protein (amino acids) breakdown along with the soaps formed during fat digestion [8,9]. Resistant starch is not digested in the gastrointestinal tract; instead, it passes relatively intact through the stomach, small intestine, and colon [30]. Therefore, it is possible that the elevated amount of RS in the grain-free diet may have resulted in fewer starch breakdown products being emptied into the small intestine. The duodenal receptors slow GER when triggered. However, if the grain-free diet had fewer breakdown products, then this process does not occur as frequently compared to the other diets. In comparison, the diets containing less RS may have induced a larger amount of starch breakdown products to enter the intestine, stimulating the receptors to slow GE.

A second possible explanation for the seemingly contradictory results may be related to the high protein/high fat background diet that the dogs received during the acclimation period two months prior to the start of the study and on days between treatments. Duodenal receptors are thought to slow GE when triggered by the breakdown of fat, protein and starch. However, the $25 \mathrm{~g}$ AvCHO portion of the grain-free diet, which contained the greatest amount of fat and protein compared to the vegan and traditional diet portions, induced the fastest GER. Research completed in rats and humans suggests that the consumption of a high protein/high fat diet for as little as 2 weeks accelerates the GER of a different high protein/high fat test diet significantly compared to a low protein/low fat test diet [31,32]. Additionally, French et al. (2007) found that CCK, a potent inhibitor of GE, usually stimulated by protein and fat entering the intestine, increased following the ingestion of a high fat diet for 2 weeks [33]. However, this did not result in a reduction of GER [33]. Together, the data from these studies suggest that subjects may be desensitized to the effects of CCK following a period of high protein/high fat consumption. It is possible that after consuming the high protein/high fat background diet for two months prior to the start of the present study, the dogs became desensitized to the effects of CCK, allowing the higher protein and fat grain-free diet to accelerate GER. Although the $25 \mathrm{~g}$ AvCHO portion of the grain-free diet provided slightly less protein and fat than the whole-grain diet which induced slower emptying, the difference in protein and fat between diets was negligible. Additionally, the slower emptying for the whole-grain diet may also be attributed to the higher RDS and lower RS and SDS as discussed above, considering the difference in starch composition was greater than the difference in fat and protein content between the whole-grain and grain-free diets. This may lend some indication as to why the ksB3 value for the grain-free diet was significantly greater than the ksв3 value for the vegan and traditional diets, which had the lowest amounts of protein and fat per $25 \mathrm{~g} \mathrm{AvCHO}$ portion compared to the grain-free diet. 
This hypothesis cannot be substantiated with certainty as the starch composition of the background diet was not analyzed, but does warrant future investigations to study the effect of dietary history and macronutrient composition on GER in dogs.

The varying levels of fiber in the test diets could have also affected the GER. Fiber naturally present in food can delay the GER of a solid meal [34]. Typically, soluble fiber has a much higher water holding capacity than insoluble dietary fiber, causing solublefiber rich foods to increase viscosity in the stomach, delaying emptying into the small intestine [35]. When comparing all treatments, the $25 \mathrm{~g} \mathrm{AvCHO}$ portion of the grain-free diet, which induced the highest GER, contained the highest amount of TDF compared to the vegan and traditional diet portions, which had the lowest amount of TDF. Additionally, the $25 \mathrm{~g} \mathrm{AvCHO}$ portion of the grain-free diet had the greatest amount of crude fiber compared to the traditional, vegan and whole grain diet portions . Total dietary fiber includes both soluble and insoluble fiber fractions, while crude fiber consists only of insoluble fiber [36]. The difference in crude fiber was larger than the difference in TDF, suggesting higher insoluble fiber in the grain-free diet, which could have resulted in less viscosity in the stomach and faster GER compared to the other treatments. In contrast, the larger amount of soluble fiber in the other diets could result in more viscosity in the stomach and consequently slower GE into the intestine; this has been previously demonstrated in human studies [37]. Less soluble fiber could have contributed to the higher ksв3 value observed for the grain-free diet. The grain-free diet had less TDF compared to the whole-grain diet; however, similar to the fat and protein content of these diets, this difference is negligible and the difference in starch profiles between the diets likely played a larger role in the observed results. This hypothesis cannot be substantiated as the test diets were not specifically analyzed for soluble and insoluble fiber fractions.

Nutrients entering the duodenum following meal ingestion activate a proximaldistal neuroendocrine loop which stimulates glucagon-like peptide-1 (GLP-1) secretion [38]. Glucagon-like peptide-1 stimulates insulin secretion and inhibits glucagon, which is responsible for raising concentrations of glucose in the blood stream [38]. As discussed above, the $25 \mathrm{~g} \mathrm{AvCHO}$ portion of the grain-free diet had greater protein and fat being broken down and entering the duodenum compared to the traditional and vegan diets, which could stimulate more GLP-1 production and therefore less glucagon, contributing to a lower PPG. This could explain why the diet with the fastest GER induced the lowest PPG. More research is warranted to better understand the relationship between GER, PPG and GLP-1 in dogs. Additionally, it may be of interest to investigate the effects that this correlation has on satiety and appetite control, as GLP-1 promotes satiety and can reduce food intake in humans [38]. This could be an important contributing factor to weight gain/obesity risk.

A limitation of this study is that the dogs only consumed a portion of each diet that provided $25 \mathrm{~g}$ of AvCHOs. This was necessary in order to concomitantly perform glycemic index testing. However, future research is necessary to investigate the effect of consumption of a full meal as this would alter the amount of nutrient digestion and absorption in the gastrointestinal tract and could subsequently affect GER.

While the data presented herein helps to improve our understanding of the effects of different diets on GER in dogs, future research investigating the effect of macronutrients on GE using a dog model is warranted. Due to the effect of starch structure on gut microbiome composition and the potential link between the microbiome and PPG $[35,39]$, it may be beneficial to explore this relationship further in dogs.

\section{Conclusions}

The results of this study indicate that the grain-free diet, which had the highest amount of RS and SDS and lowest amount of TS and RDS, induced more overall GE at a more rapid rate in Siberian Huskies compared to the traditional, vegan and whole-grain diets and the glucose control. Authors hypothesize that these findings may be due to the 
differences in macronutrient content of the test foods, including the composition of the starch sources. The fat and protein content of the background diet and test diets, as well as the fiber content of the test diets may have also contributed to the observed results. It may be of interest to further explore the relationship between GER and PPG, specifically in regard to CCK, GLP-1, satiety and appetite control. This research may be paramount in determining how to prevent weight gain and ultimately reduce the prevalence of obesity in companion animals.

Author Contributions: Conceptualization, JPC, AKS, JLA, DR, AV; methodology, JPC, AKS, JLA, DR, AV; formal analysis, TLR, AR, JPC; investigation, AR, AKS, JLA, AV; resources, JPC, DR; writing - original draft preparation, TLR, AR; writing - review and editing, AR, JPC, AKS, JLA, DR, AV; supervision, AKS, JLA, AV; project administration, $\mathrm{AR}$, JLA, AV; funding acquisition, JLA, AV. All authors have read and agreed to the published version of the manuscript.

Funding: This study was funded by a Natural Science \& Engineering Research Council Collaborative Research Development grant (\#CRDPJ488705-15) in partnership with PPN Limited Partnership (Petcurean). Support for A.R. was provided by a Mitacs Accelerate grant (IT09138) in collaboration with PPN Limited Partnership.

Institutional Review Board Statement: The experimental protocol for this research was approved by the University of Guelph Animal Care Committee (AUP\#3650) and in accordance with national and institutional guidelines for the care and use of animals.

Data Availability Statement: The data presented in this study are available on request from the corresponding author.

Acknowledgments: We would like to thank all student volunteers for all their help with animal handling and blood collections, as well as Dr. Sarah Dodd and Deirdre Stuart for assisting with catheter placement. Additionally, we would like to extend a special thank you to the owners of the sled dogs.

Conflicts of Interest: J.L.A. was a paid employee of PPN Limited Partnership (Petcurean) at the time of the study and is currently employed by ADM. AV is the Royal Canin Veterinary Diets Endowed Chair in Canine and Feline Clinical Nutrition and serves on the Health and Nutrition Advisory Board for Vetdiet. AV and AKS receive honoraria and research funding from various pet food manufacturers and ingredient suppliers. AR has participated in paid internships and engage-ments with various pet food companies within Canada. The Ontario Veterinary College received funding from Purina Pro Plan Veterinary Diets to support a nutrition technician. The remaining authors declare no real or perceived conflicts of interest.

\section{References}

1. Kealy, R.D.; Lawler, D.F.; Ballam, J.M.; Mantz, S.L.; Biery, D.N.; Greeley, E.H.; Lust, G.; Segre, M.; Smith, G.K.; Stowe, H.D. Effects of diet restriction on life span and age-related changes in dogs. J. Am. Vet. Med. Assoc. 2002, 220, 1315-1320.

2. Kipperman, B.S.; German, A.J. The responsibility of veterinarians to address companion animal obesity. Animals 2018, 8, 143.

3. German, A.J. The growing problem of obesity in dogs and cats. J. Nutr. 2006, 136, 1940S-1946S.

4. Hill, J.O.; Wyatt, H.R.; Peters, J.C. Energy balance and obesity. Circulation 2012, 126, 126-132.

5. Marathe, C.S.; Rayner, C.K.; Jones, K.L.; Horowitz, M. Relationships between gastric emptying, postprandial glycemia, and incretin hormones. Diabetes Care 2013, 36, 1396-1405.

6. Tosetti, C.; Corinaldesi, R.; Stanghellini, V.; Pasquali, R.; Corbelli, C.; Zoccoli, G.; Di Febo, G.; Monetti, N.; Barbara, L. Gastric emptying of solids in morbid obesity. Int. J. Obes. Relat. Metab. Disord. 1996, 20, 200-205.

7. Bourreau, J.; Hernot, D.; Bailhache, E.; Weber, M.; Ferchaud, V.; Biourge, V.; Martin, L.; Dumon, H.; Nguyen, P. Gastric emptying rate is inversely related to body weight in dog breeds of different sizes. J. Nutr. 2004, 134, 2039S-2041S. 
8. Hunt, J.N.; Stubbs, D.F. The volume and energy content of meals as determinants of gastric emptying. J. Physiol. 1975, 245, 209225.

9. Elias, E.; Gibson, G.J.; Greenwood, L.F.; Hunt, J.N.; Tripp, J.H. The slowing of gastric emptying by monosaccharides and disaccharides in test meals. J. Physiol. 1968, 194, 317-326.

10. Abd El-Khalek, E.; Janssens, G.P.J. Effect of extrusion processing on starch gelatinisation and performance in poultry. Worlds. Poult. Sci. J. 2010, 66, 53-63.

11. Roberts, M.T.; Bermingham, E.N.; Cave, N.J.; Young, W.; McKenzie, C.M.; Thomas, D.G. Macronutrient intake of dogs, selfselecting diets varying in composition offered ad libitum. J. Anim. Physiol. Anim. Nutr. (Berl). 2018, 102, 568-575.

12. Englyst, K.N.; Liu, S.; Englyst, H.N. Nutritional characterization and measurement of dietary carbohydrates. Eur. J. Clin. Nutr. 2007, 61, 19S-39S.

13. Nelson, D., and M. Cox. Lehninger Principles of Biochemistry, 5th ed; WH Freeman. New York, 2008.

14. Biliaderis, C. G. The structure and interactions of starch with food constituents. Can. J. Physiol. Pharmacol. 1991, 69, 60-78.

15. Joshi, M.; Aldred, P.; McKnight, S.; Panozzo, J.F.; Kasapis, S.; Adhikari, R.; Adhikari, B. Physicochemical and functional characteristics of lentil starch. Carbohydr. Polym. 2013, 92, 1484-1496.

16. Thakur, R.; Pristijono, P.; Scarlett, C.J.; Bowyer, M.; Singh, S.P.; Vuong, Q. V. Starch-based films: Major factors affecting their properties. Int. J. Biol. Macromol. 2019, 132, 1079-1089.

17. Hoover, R.; Sosulski, F.W. Composition, structure, functionality, and chemical modification of legume starches: A review. Can. J. Physiol. Pharmacol. 1991, 69, 79-92.

18. Englyst, H. N., S. M. Kingman, and J. H. Cummings. Classification and measurement of nutritionally important starch fractions. Eur. J. Clin. Nutr. 1992, 46, 33S-50S.

19. Jenkins, D.J.A.; Wolever, T.M.S.; Taylor, R.H.; Barker, H.; Fielden, H.; Baldwin, J.M.; Bowling, A.C.; Newman, H.C.; Goff, D. V. Glycemic index of foods: A physiological basis for carbohydrate exchange. Am. J. Clin. Nutr. 1981, 34, 362-366.

20. Stahel, P.; Cant, J.P.; MacPherson, J.A.R.; Berends, H.; Steele, M.A. A mechanistic model of intermittent gastric emptying and Glucose-Insulin dynamics following a meal containing milk components. PLoS One 2016, 11.

21. Glerup, H.; Bluhme, H.; Villadsen, G.E.; Rasmussen, K.; Ejskjaer, N.; Dahlerup, J.F. Gastric emptying: A comparison of three methods. Scand. J. Gastroenterol. 2007, 42, 1182-1186.

22. Ehsani-Kheradgerdi, A.; Sharifi, K.; Mohri, M.; Grünberg, W. Evaluation of a modified acetaminophen absorption test to estimate the abomasal emptying rate in holstein-friesian heifers. Am. J. Vet. Res. 2011, 72, 1600-1606.

23. Rankovic, A.; Adolphe, J.L.; Dan Ramdath, D.; Shoveller, A.K.; Verbrugghe, A. Glycemic response in nonracing sled dogs fed single starch ingredients and commercial extruded dog foods with different carbohydrate sources. J. Anim. Sci. 2020, 98.

24. Laflamme, D. Development and validation of a body condition score system for dogs. Canine Pract. 1997, $22,10-15$.

25. National Research Council. Nutrient Requirements of Dogs and Cats; National Academy of Sciences: Washington, DC, USA, 2006.

26. Hall, J.A.; Melendez, L.D.; Jewell, D.E. Using Gross Energy Improves Metabolizable Energy Predictive Equations for Pet Foods Whereas Undigested Protein and Fiber Content. PLOS ONE 2013, 8.

27. Brummer, Y.; Kaviani, M.; Tosh, S.M. Structural and functional characteristics of dietary fibre in beans, lentils, peas and chickpeas. Food Res. Int. 2015, 67, 117-125.

28. Ramdath, D.D.; Liu, Q.; Donner, E.; Hawke, A.; Kalinga, D.; Winberg, J.; Wolever, T.M.S. Investigating the relationship between lentil carbohydrate fractions and in vivo postprandial blood glucose response by use of the natural variation in starch fractions among 20 lentil varieties. Food Funct. 2017, 8, 3783-3791.

29. Hasek, L.Y.; Phillips, R.J.; Hayes, A.M.R.; Kinzig, K.; Zhang, G.; Powley, T.L.; Hamaker, B.R. Carbohydrates designed with different digestion rates modulate gastric emptying response in rats. Int. J. Food Sci. Nutr. 2020, 71, 839-844.

30. Birt, D.F.; Boylston, T.; Hendrich, S.; Jane, J.L.; Hollis, J.; Li, L.; McClelland, J.; Moore, S.; Phillips, G.J.; Rowling, M.; et al. Resistant starch: Promise for improving human health. Adv. Nutr. 2013, 4, 587-601.

31. Shi, G.; Leray, V.; Scarpignato, C.; Bentouimou, N.; Bruley Des Varannes, S.; Cherbut, C.; Galmiche, J.P. Specific adaptation of gastric emptying to diets with differing protein content in the rat: Is endogenous cholecystokinin implicated? Gut 1997, 41, 612618.

32. Castiglione, K.E.; Read, N.W.; French, S.J. Adaptation to high-fat diet accelerates emptying of fat but not carbohydrate test meals in humans. Am. J. Physiol. Regul. Integr. Comp. Physiol. 2002, 282.

33. French, S.J.; Murray, B.; Rumsey, R.D.E.; Fadzlin, R.; Read, N.W. Adaptation to high-fat diets: effects on eating behaviour and plasma cholecystokinin. Br. J. Nutr. 1995, 73, 179-189.

34. Tambascia, M.A.; Malerbi, D.A.C.; Eliaschewitz, F.G. Influence of gastric emptying on the control of postprandial glycemia: physiology and therapeutic implications. Einstein (Sao Paulo) 2014, 12, 251-253.

35. Søndertoft, N.B.; Vogt, J.K.; Arumugam, M.; Kristensen, M.; Gøbel, R.J.; Fan, Y.; Lyu, L.; Bahl, M.I.; Eriksen, C.; Ängquist, L.; et al. The intestinal microbiome is a co-determinant of the postprandial plasma glucose response. PLoS One 2020, 15.

36. Institute of Medicine (US) Panel on the Definition of Dietary Fiber and the Standing Committee on the Scientific Evaluation of Dietary Reference Intakes. Appendix C, Development and Evolution of Methods Used to Extract and Measure Dietary Fiber. In Dietary Reference Intakes Proposed Definition of Dietary Fiber.; National Academies Press (US): Washington, DC, USA, 2001.

37. Holt, S.; Carter, D.C.; Tothill, P.; Heading, R.C.; Prescott, L.F. Effect of gel fibre on gastric emptying and absorption of glucose and paracetamol. Lancet 1979, 313, 636-639.

38. Gutzwiller, J.P.; Drewe, J.; Göke, B.; Schmidt, H.; Rohrer, B.; Lareida, J.; Beglinger, C. Glucagon-like peptide-1 promotes satiety and reduces food intake in patients with diabetes mellitus type 2. Am. J. Physiol. Regul. Integr. Comp. Physiol. $1999,276$. 
39. Warren, F.J.; Fukuma, N.M.; Mikkelsen, D.; Flanagan, B.M.; Williams, B.A.; Lisle, A.T.; Ó Cuív, P.; Morrison, M.; Gidley, M.J. Food Starch Structure Impacts Gut Microbiome Composition. mSphere 2018, 3. 